\title{
On the Magnetic Fields of Galaxies
}

\author{
R.R. Andreasyan * \\ Byurakan Astrophysical Observatory NAS Armenia, Byurakan, Aragatsotn prov., 0213, Armenia
}

\begin{abstract}
We bring results of some our investigations of magnetic field of our Galaxy and extragalactic radio sources. For the study were used data of Faraday rotation of pulsars and extragalactic radio sources as well as data of physical and morphological properties of more than 500 radio galaxies of different morphological classes.
\end{abstract}

Keywords: Magnetic field, Radio-galaxy, Active Galaxies

\section{Introduction}

The study of the magnetic fields of galaxies and, in particular, of our Galaxy has a great importance for explaining many dynamical and active processes taking place in these objects. The presence of the magnetic field of the Galaxy can explain the transportation of cosmic rays through the interstellar medium, as well as synchrotron background radiation in the Galaxy (Fermi, 1949). The considering the role of the magnetic field in the process of star formation has a great importance in galaxies. All of this show that it is fundamental to understand the strength and direction of the magnetic field in the ISM, as well as its morphology across the entire Galaxy.

\section{The magnetic field of the Galaxy}

The magnetic field of our Galaxy was studied using observational data of various types, such as interstellar polarization of starlight (more than 9000 stars); Zeeman splitting of spectral lines of HI and different molecules in the radio range that provides detailed information about the line-of-sight magnetic field in the ISM. But the most effective method for the study of Galactic magnetic field is based on the use of data on Faraday rotation of polarized radio emission of extragalactic radio sources (now available for about 40000 objects) and pulsars (more than 1200). It is well known that pulsars, for which numerous and diverse observational data were obtained, can be considered probes for studying the interstellar medium. In particular, data on dispersion measures (DM), which practically are known for all known pulsars, and about measures of Faraday rotation (RM) are very important for studying the magnetic field of the Galaxy. These data are directly derived from observations of pulsars. Theoretically they are expressed by the electron density $\mathrm{n}_{e}$ in the interstellar medium through which the polarized radio emission of the pulsar passes and the projection of the magnetic field $\mathrm{B}_{L}$ (in Gauss) in this medium, using the following formulas:

$$
\begin{gathered}
D M=\int n_{e} d L \\
R M=\alpha \int n_{e} B_{L} d L,\left(\alpha=8.1 * 10^{5}\right)
\end{gathered}
$$

In these formulas, integration is carried out over the entire traversed path of radiation (L in parsecs) from the pulsar to the observer. If the sign of RM is positive, it means that projection of the magnetic field $\mathrm{B}_{L}$ is directed to observer. Formula 1 makes it possible to determine the distance of a pulsar with the

*randrasy@bao.sci.am 
known electron density distribution in the Galaxy, and formula 2 together with formula 1 makes possible to determine the average component of the tension of interstellar magnetic field $\left[\mathrm{B}_{L}\right]$ on the line of sight in micro gauss $(\mu G)$.

$$
\left[B_{L}\right]=\frac{1}{\alpha} \frac{R M}{D M}=1.23 \frac{R M}{D M}
$$

All of these data were used to study the structure and magnitude of the magnetic field of the Galaxy, since the seventies of the last century, when the rotation measures and other data were known for only a few tens of objects. As the amount of important data increases, more detailed studies have been carried out and various models have been proposed for the plane component of the Galactic magnetic field, as well as for the magnetic field in the Halo of the Galaxy. Here we present some of the results that we obtained in this area.

Andreasyan \& Makarov (1989a) used the data of 185 RM data of pulsars for the study of magnetic field in the plan of Galaxy. Was construct the map of RM distribution in the galactic plan.

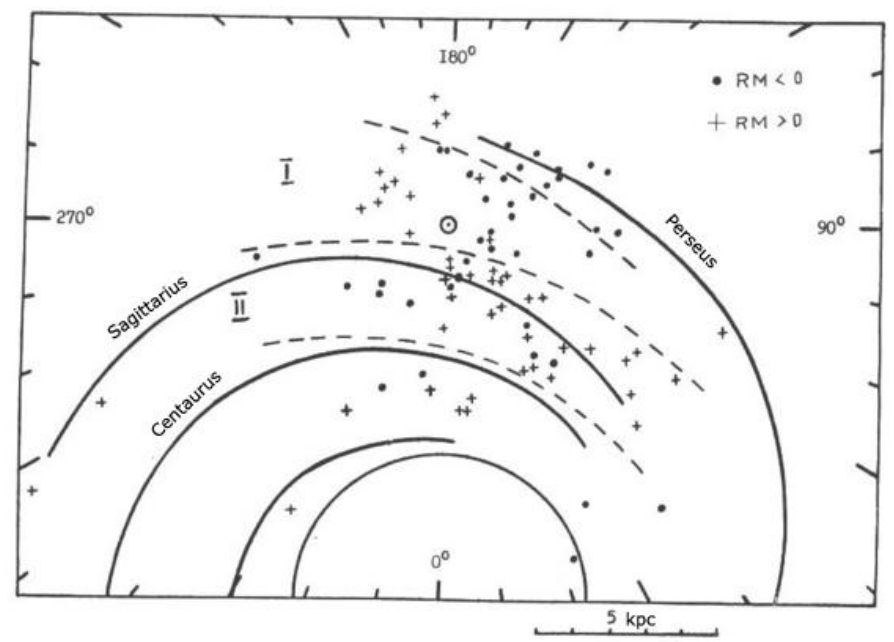

Figure 1. Distribution of RM signs for pulsars in the \pm 400 pc Galactic layer.

There are two well-defined areas in the picture. In the first area I (the local Orion arm and Perseus arm area) the magnetic field has anti clockwise direction. In the area II the magnetic field has opposite clockwise direction. It means that there is an inversion of the direction of magnetic field when passing from one spiral arm of the Galaxy to another.

Andreasyan \& Makarov (1989b) for the study of Galactic magnetic field used star light interstellar polarization data of 7500 objects. In fig. it is shown a distribution of averaged star light polarization in the layer of \pm 250 parsec near the Galactic plan.

From the picture evidences in favor of the presently accepted model of the magnetic field of the Galaxy were obtained. The magnetic fields in the Galaxy plane are mostly concentrated in the spiral arms and are directed along the axes of the corresponding arms.

Our investigation confirms the model of the two-component magnetic field of our Galaxy proposed in Andreasyan \& Makarov (1989a). For the first time it was shown that the data on the rotation measures of pulsars and extragalactic radio sources are in good agreement with the model when the magnetic field of the plane component of the spiral arms is embedded in the magnetic field of the galactic halo with dipole configuration, which is deformed due to the differential rotation of the Galaxy.

In Andreasyan et al. (2011) a method is introduced for constructing two-color maps. Using the formulas 1 and 2 we obtain

$$
\begin{gathered}
B_{L}(D M)=\frac{1}{\alpha} \frac{d R M}{d D M} \\
B_{L}(R) n_{e}(R)=\frac{1}{\alpha} \frac{d R M}{d(R)}
\end{gathered}
$$

where $\mathrm{B}_{L}(\mathrm{DM})$ is the line of sight component of magnetic field strength at the point with a given value of 
$\mathrm{DM}, \mathrm{B}_{L}(\mathrm{R})$ is the line of sight component of magnetic field strength at the point with a distance $\mathrm{R}$ from the Sun. It means, that using the RM-DM and RM-R dependences for a given direction, it is possible to find $\mathrm{B}_{L}(\mathrm{DM})$ for each value of $\mathrm{DM}$, and $\mathrm{B}_{L}(\mathrm{R}) \mathrm{n}_{e}(\mathrm{R})$ for each value of $\mathrm{R}$. We can find the RM-DM and RM-R dependences for all directions in the plan of Galaxy using averaging procedure. In fact we are solving the inverse problem to construct 2-dimensional maps for plane component of Galactic magnetic field with coordinates $(1 ; \mathrm{DM})$, or $(1 ; \mathrm{R})$. Using the data of $\mathrm{RM}, \mathrm{DM}$ and the distance of pulsars, we constructed a two-color map of the plane component of the magnetic field of the Galaxy, on which large-scale regions with regular magnetic fields, approximately corresponding to the spiral structure of the Galaxy, were clearly distinguished. Some results of calculations are given on the maps of $\mathrm{B}_{L}(\mathrm{R}) \mathrm{n}_{e}(\mathrm{R})$ and $\mathrm{B}_{L}(\mathrm{DM})$.

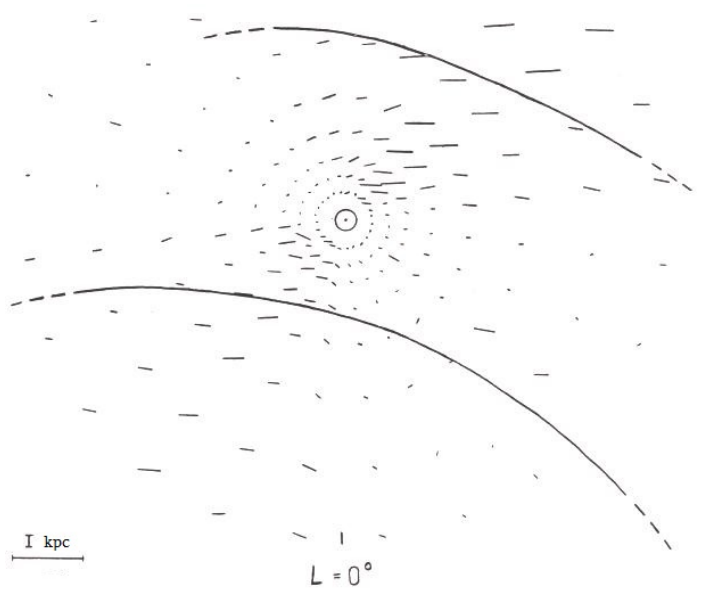

Figure 2. Star light interstellar polarization parameters of space groups of stars in the \pm 250 pc layer. The directions of the spiral arms of Perseus and Sagittarius-Carina are also shown.
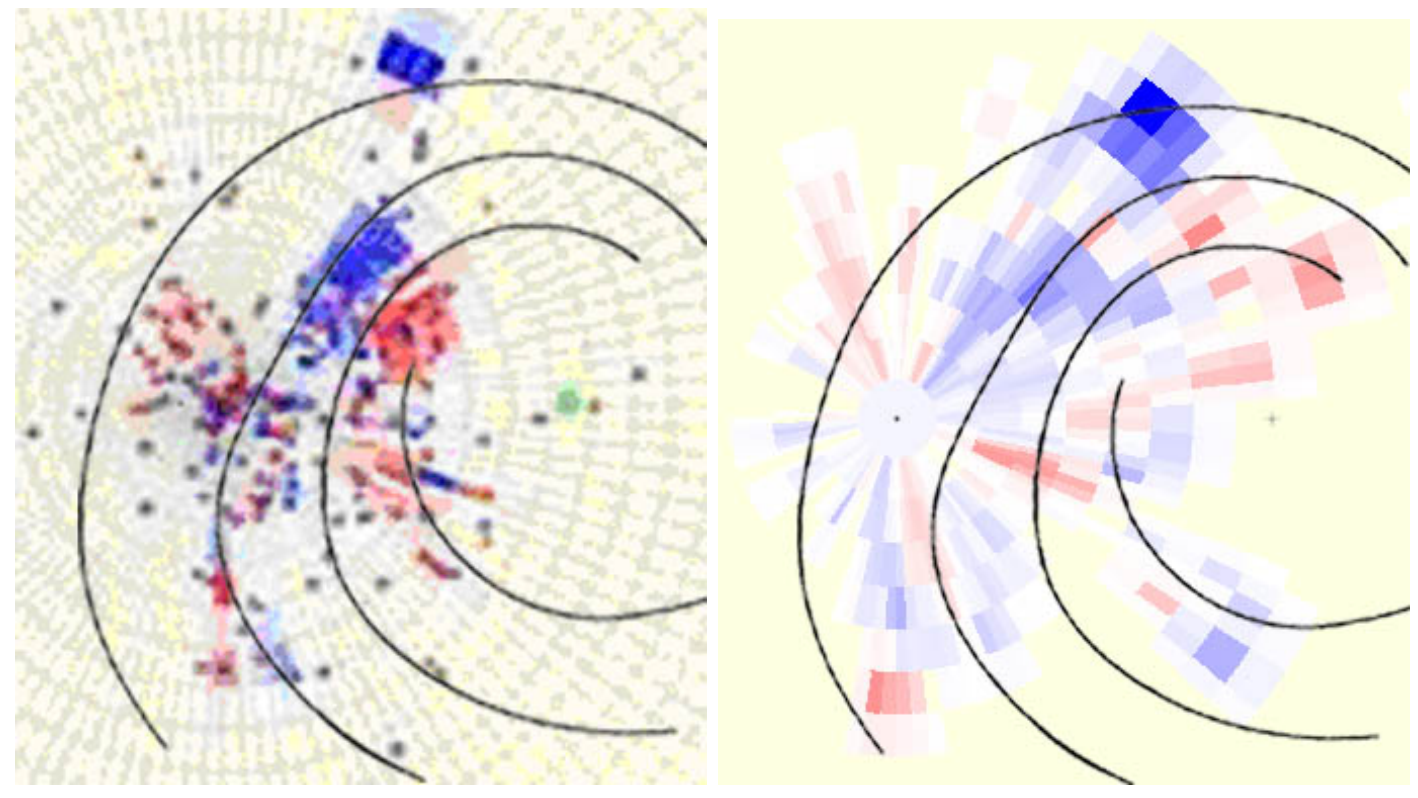

Figure 3. The distributions of $\mathrm{B}_{L}(\mathrm{R}) \mathrm{n}_{e}(\mathrm{R})$ (with pulsars) in coordinates $(1 ; \mathrm{R})$ (the left picture), and $\mathrm{B}_{L}(\mathrm{DM})$ in coordinates $(1 ; \mathrm{DM})$ (right picture) with the spiral arms of the Galaxy, red color for magnetic field directed to observer and blue color for the field directed from the observer. The intensity of the color shows the strength of the field) for the plane component of the magnetic field of our galaxy in (R, l) and (DM, l) coordinates. The sun is on the distance of $8.5 \mathrm{kpc}$ to the left side from the Galactic center.

Using these pictures, identifying some details on two distributions it is possible to find the magnetic field strength in every point of Galactic plan.

In Andreasyan et al. (2020) is modeled reversals of Galactic magnetic field using the so-called no-z approximation based on the fact that the galactic disk is sufficiently thin. The magnetic field generation that exhibit both single and double sign changes with distance from the center is observed. There are 
numerous arguments in favor of the fact that the magnetic field exhibits so-called reversals associated with a direction change from one region to another. A study of the pulsar distribution with large Faraday rotation measures $\left(R M>300 \mathrm{rad} / \mathrm{m}^{2}\right)$ was presented. The results indicate that there is a region of the Galaxy (the galactocentric ring inside $\approx 5 \mathrm{kpc}$ and $7 \mathrm{kpc}$ ), in which the magnetic field is oriented counterclockwise.

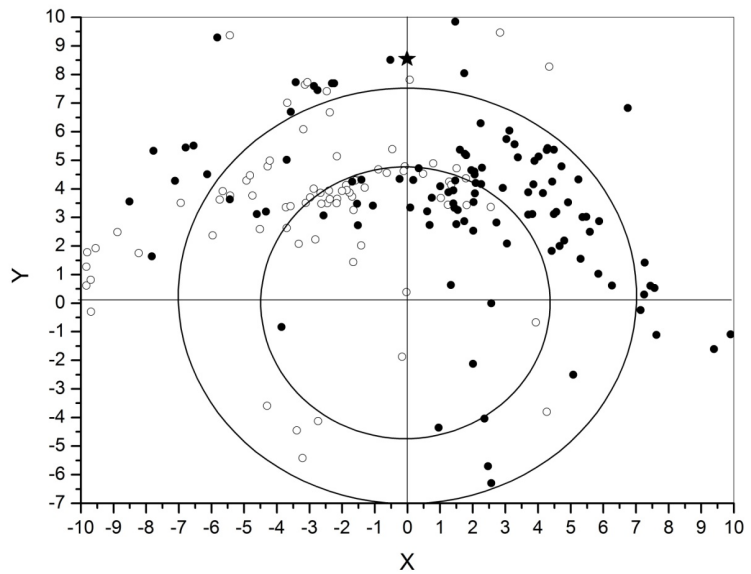

Figure 4. Distribution of rotation measures of pulsars in the plane of the Galaxy.

On the Fig. 4 black circles denote pulsars in $R M>300 \mathrm{rad} / \mathrm{m}^{2}$ (the projection of the magnetic field on the line of sight is directed toward the observer), white circles - in which $R M<-300 \mathrm{rad} / \mathrm{m}^{2}$ (the projection of the magnetic field is directed from the observer). At the ring edges $(\approx 5 \mathrm{kpc}$ and $7 \mathrm{kpc})$ the magnetic field reverses its direction. These results are in close agreement with our theoretical concepts. As we see from the observations it can be studied the configuration and strength of Galactic magnetic field. The generation of these fields is explained by the dynamo mechanism associated with motions of the interstellar medium in appropriate objects. The growth of magnetic fields is exponential and these become stabilized when the equipartition of energy between magnetic fields and turbulent motions is reached. But for starting this generation mechanism, some initial "seed" magnetic fields are necessary, and these fields are not explained within the dynamo theory. The so-called Biermann battery mechanism is very good for the formation of "seed" magnetic fields. If we have an outflow of plasma in rotating interstellar medium of galaxies, then because of the large differences between scattering time of expanding electrons and protons with the rotating medium, in every point of galaxy the rotation velocity of scattered electrons and protons will correspond to the rotation velocity of their last scattering point and will be different. In the result of forming of circular electric currents in galaxies evaluates dipolar magnetic fields. Some simple estimates of the strength of such a field in active galaxies were found in our earlier works. Now we have constructed a self-consistent model and derived an integral equation, which permits both to determine the order of magnitude of the initial magnetic field and to study in detail its spatial structure. These magnetic fields evaluate to large-scale magnetic fields by Dinamo mechanisms. In this way can be generated large-scale dipolar magnetic fields.

\section{Extragalctic radio sources}

The dipolar configuration of large-scale magnetic fields of active galaxies was the main suggestion for the mechanism of the formation and evolution of extragalactic radio sources (Andreasyan, 1983). In frame of this mechanism extragalactic radio sources are formed from relativistic plasma clouds, ejected from the central part of the optical galaxy and moving in large-scale dipolar magnetic field of parent galaxy. The behaviors of relativistic plasma cloud, ejected in the direction of the dipole axis, depends on the ratio $\mathrm{Q}$ of the kinetic energy density of the plasma to the magnetic field energy density,

- If the ratio $\mathrm{Q}$ is greater than unity the clouds of charged particles, expanding, travels large distances from the optical galaxy, carrying with them the magnetic field lines. Similar features we observe in extragalactic radio sources of FRII classes (Fanaroff \& Riley (1974), edge brightened, more powerful objects).

- If the ratio Q of energy densities is less than unity, the charged particles will move along the field lines of the dipole magnetic field of the galaxy. In this case will be evaluate extragalactic radio sources of 


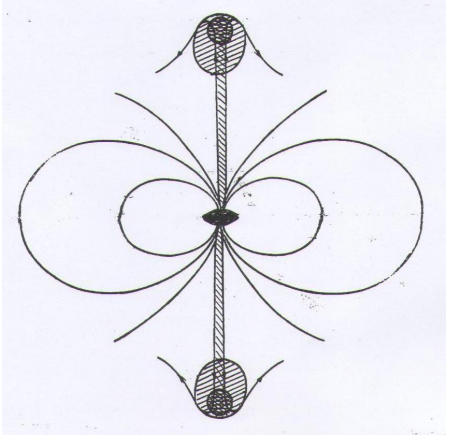

Figure 5. The ratio $\mathrm{Q}$ is greater than unity $(Q>1)$.

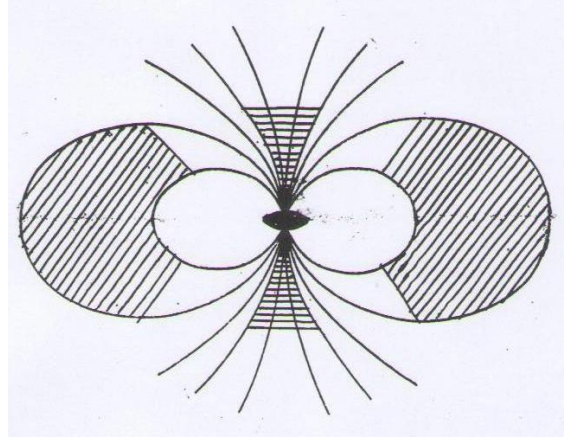

Figure 6. The ratio $\mathrm{Q}$ is less than unity $(Q<1)$.

In Andreasyan $(1984,1985)$ parallel to the well-known Fanaroff-Riley classification we bring also a simple classification of extragalactic radio sources by the elongation parameter $\mathrm{K}$ (the ratio of the largest dimension of radio image to the perpendicular dimension).

\subsection{Observational data}

For the study it was used data for 267 nearby radio galaxies identified with elliptical galaxies brighter than $18^{t} h$ magnitude (sample1) (Andreasyan \& Sol, 1999), and 280 extragalactic radio sources with known position angles between the integrated intrinsic radio polarization and radio axes (sample 2) (Andreasyan et al., 2002). Here there are some results (physical and morphological differences in different classes of extragalactic radio sources) obtained in our study (Andreasyan \& Sol, 1999, 2000, Andreasyan et al., 2002, 2013).

\subsection{The correlation of radio axis with the optical axis in nearby radio galaxies}

Data from sample of 267 nearby radio galaxies were used to study the correlations of radio axes with the optical axes of parent galaxies. Were constructed histograms separately for radio galaxies classified by elongation and for radio galaxies with FR classes. On the figures the difference between the radio and optical position angles (dPA) is laid out along the horizontal axis and the number of radio galaxies along the vertical axis.
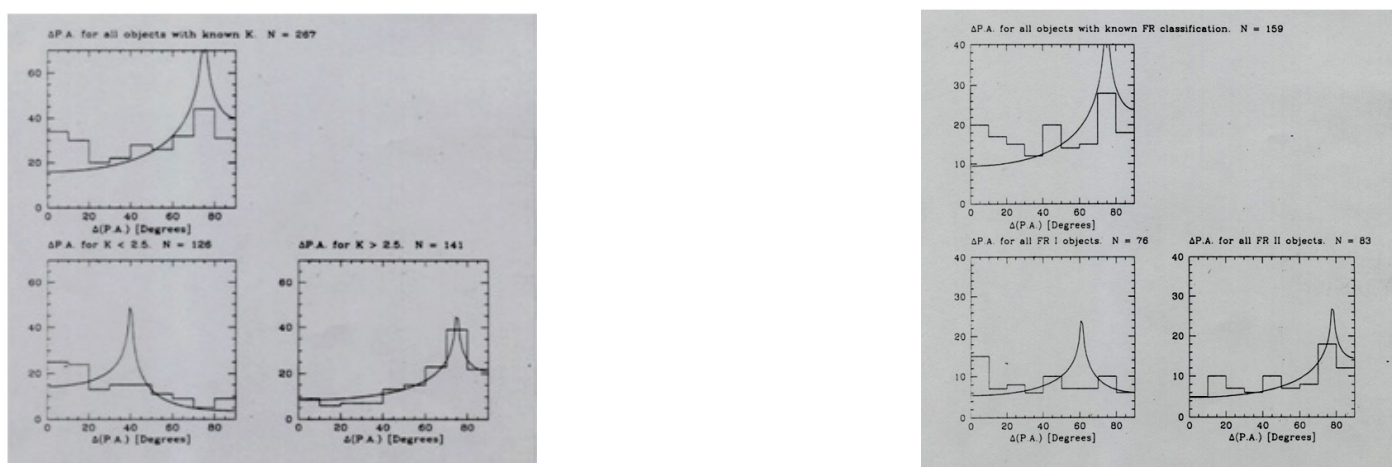

Figure 7. The distribution of (dPA) for K classes. Figure 8. The distribution of (dPA) for FR classes.

From the figures we found, that more elongated and FRII type radio galaxies are in most cases directed as minor axes or rotation axes of host elliptical galaxies, while the less elongated and FRI ones are directed perpendicular to these axes. This result is in a good agreement with our conclusions.

\subsection{The ellipticity of elliptical galaxies identified with the different types of extragalac- tic radio sources}

In the Sample 1 we have data of the optical ellipticity (E) of 154 elliptical galaxies. For all of them we have the elongation parameters $\mathrm{K}$ and for 95 - the Fanaroff-Riley classes. We use this data to study the 
distribution of ellipticities of parent optical galaxies for different FR types and for different classes of our K classification.

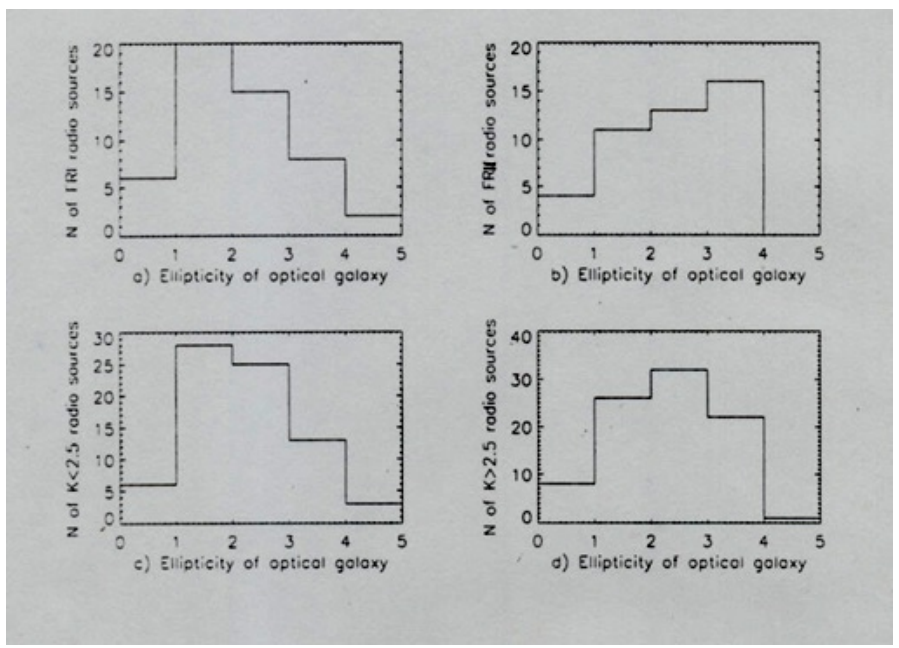

Figure 9. The ellipticities of optical parent galaxies for different FR types and for different classes of $\mathrm{K}$ classification.

From the Fig. 9 it is clear that the host elliptical galaxies of less elongated extragalactic radio sources and radio sources of $\mathrm{FRI}$ type have less ellipticity ( $\mathrm{E} \approx 1$ to 2 ) than these of radio sources of large elongation and radio galaxies FRII type ( $\mathrm{E} \approx 3$ to 4 ). The fact that host elliptical galaxies of FRI type radio sources have less ellipticities can be explained in two ways: It is primordial and in some way is responsible for the formation of FRI types, or it is from the orientation effect. In both cases, the fact of different ellipticities of different FR types is interesting for the understanding of formation of radio sources.

\subsection{The correlation of the radio polarization angle with the radio axes of extragalactic radio sources}

The data of 280 extragalactic radio sources of sample 2 were used for the study of distribution of angles $\Delta(\mathrm{PA})$ between directions of integrated intrinsic radio polarization and the major axes for different type radio sources, classified by their elongation and FR classification. The histograms of angles between radio and polarization axes are shown on pictures.
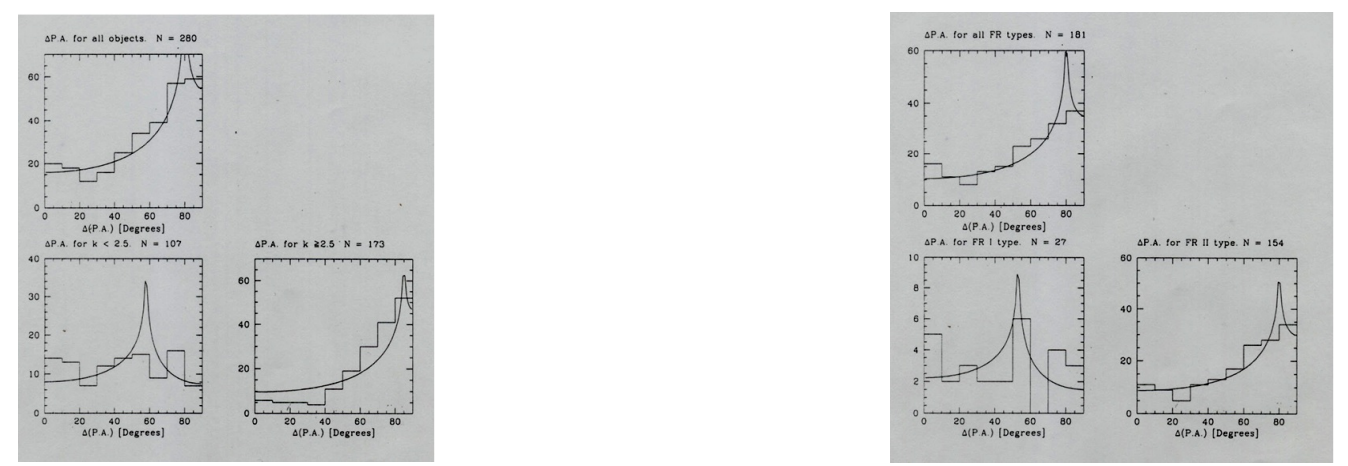

Figure 10. The distribution of $\Delta(\mathrm{PA})$ for $\mathrm{K}$ classes. Figure 11. The distribution of $\Delta(\mathrm{PA})$ for FR classes.

We see that for elongated and FRII sources, their intrinsic integrated polarization is perpendicular to their major radio axes. As the magnetic fields in optically thin synchrotron radio sources are perpendicular to the polarization of electric vector, the main result of this study is that integrated magnetic fields can be described as intrinsically aligned with major radio axes for elongated and FRII radio sources, while they are not correlated with radio axes for stocky and FRI radio sources. 


\subsection{Distribution of the elongation parameter of the extragalactic radio sources for the different FR classes}

Here we study the distribution of the elongations of the radio images for radio sources in the two FR classes. Data from samples 1,2 , are used to construct the distribution functions $f(\mathrm{~K})$ of the elongation parameter $\mathrm{K}$ which are shown in the following figures.

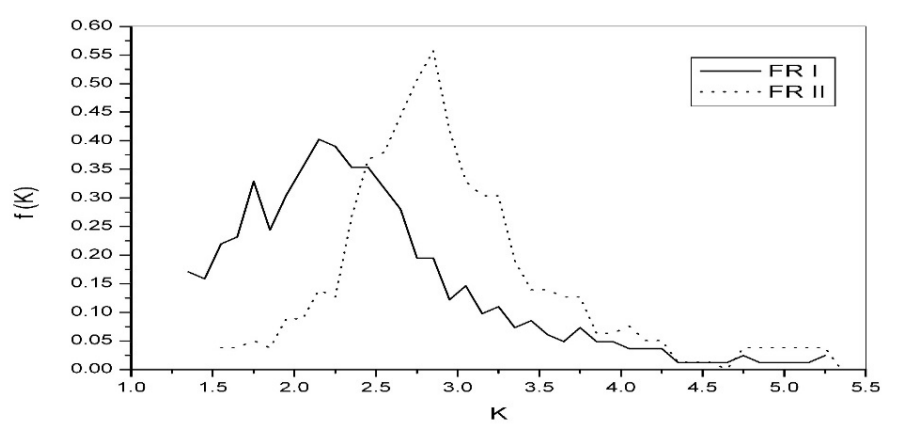

Figure 12. The distribution functions of the parameter K (using 82 FRI radio sources and 79 FRII radio sources).

These figures show that the peak of the distribution of FRII radio sources is at about $\mathrm{K} \approx 3$, while for the FRI sources the peak is at roughly $\mathrm{K} \approx 2$ that is, the FRII radio sources are, on the average, more elongated than the FRI sources. We believe that another important difference between the distributions of FRI and FRII extragalactic radio sources is that the FRI distribution has two peaks. 


\section{Acknowledgements}

The work was supported by the Science Committee of RA, in the frames of the research project No. 21AG-1C053 "Revelation of the early stages of galaxy evolution by means of multiwavelength study of active galaxies".

\section{References}

Andreasyan R. R., 1983, Astrophysics, 19, 245

Andreasyan R. R., 1984, Astrophysics, 21, 409

Andreasyan R. R., 1985, Astrophysics, 23, 404

Andreasyan R. R., Makarov A. N., 1988, Astrophysics, 28, 247

Andreasyan R. R., Makarov A. N., 1989a, Astrophysics, 30, 101

Andreasyan R. R., Makarov A. N., 1989b, Astrophysics, 31, 560

Andreasyan R. R., Sol H., 1999, Astrophysics, 42, 275

Andreasyan R. R., Sol H., 2000, Astrophysics, 43, 413

Andreasyan R. R., Appl S., Sol H., 2002, Astrophysics, 45, 198

Andreasyan R. R., Balayan S. K., Movsesyan V. H., 2011, Astrophysics, 54, 177

Andreasyan R. R., Hovhannisyan M. A., Paronyan G. M., Abrahamyan H. V., 2013, Astrophysics, 56, 382

Andreasyan R. R., Mikhailov E. A., Andreasyan H. R., 2020, Astronomy Reports, 64, 189

Fanaroff B. L., Riley J. M., 1974, MNRAS, 167, 31

Fermi E., 1949, PhysRev, 75, 1169 